Bio-grafia Escritos sobre La Biología y su Enseñanza.

Edición Extra-Ordinaria. ISSN 2027-1034 P. p 87 - 94

Memorias del VII Encuentro Nacional de Experiencias en la Enseñanza de la

Biología y la Educación Ambiental y II Congreso Nacional de Investigación en la Enseñanza de la Biología

\title{
LA DIMENSIÓN AMBIENTAL: \\ “UNA INCLUSIÓN NECESARIA EN LA FORMACIÓN DE PROFESORES DE QUÍMICA"
}

\section{THE ENVIRONMENTAL DIMENSION: "A NECESSARY INCLUSION FORMING IN CHEMISTRY TEACHERS"}

\author{
Diana L. Parga Lozano ${ }^{1}$ \\ William M. Mora $\mathbf{P}^{2}$ \\ Yiny P. Cárdenas R. ${ }^{3}$
}

\section{Resumen}

La forma en que esta incluida la dimensión ambiental en el programa de formación de profesores de química de la Universidad Pedagógica Nacional de Colombía, se convierte en el horizonte de la presente investigación. Es por esto que se han diseñado instrumentos enfocados desde una matriz de análisis que abarcó los diferentes tipos de conocimiento planteados por Mora y Parga (2008) en lo que se conoce como el Conocimiento Didáctico del Contenido Curricular (CDCC) que es un conocimiento que emerge de la interacción e integración del conocimiento disciplinar, el Histórico-Epistemológico, Conocimiento del Contexto Escolar y Conocimiento Psicopedagógico; cada uno de éstos considerados esenciales para la inclusión de los componentes de la dimensión ambiental planteados por Caride y Meira (2001) quien establece como pilares de la Educación Ambiental, las dimensiones económica, política, social, cultural, ética y la ecológica.

Es así que se tuvo en cuenta para su determinación documentos tales como el Plan de Desarrollo Institucional 2009-2013, Misión y Visión tanto de la Facultad de Ciencia y Tecnología, como el Departamento de Química, por otro lado se analizo los syllabus de los espacios académicos ofertados por el programa curricular donde se determino los que posiblemente tenían inmersa la dimensión ambiental en alguno de los componentes que se citan a lo largo de la investigación. Estos resultados permitiran el diseño de una propuesta curricular que incluya la dimensión ambiental en la formación de profesores de química.

Palabras Clave: Dimensión Ambiental, Conocimiento Didáctico del Contenido Curricular, Formación de profesores.

\footnotetext{
${ }^{1}$ Profesora Universidad Pedagógica Nacional, dparga@pedagogica.edu.co.

${ }^{2}$ Profesor Universidad Distrital Francisco José de Caldas, wmora@udistrital.edu.co.

${ }^{3}$ Estudiante Maestría en Docencia de la Química Universidad Pedagógica Nacional, yipacaro22@gmail.com
} 
Bio-grafía Escritos sobre la Biología y su Enseñanza.

Edición Extra-Ordinaria. ISSN 2027-1034 P.p 87 - 94

Memorias del VII Encuentro Nacional de Experiencias en la Enseñanza de la

Biología y la Educación Ambiental y II Congreso Nacional de Investigación en la Enseñanza de la Biología

\section{Abstract}

The way that the environmental dimension is included in the training program for teachers of chemistry National Pedagogical University of Colombia, becomes the horizon of the present investigation. This is why we have designed instruments focused from an analysis matrix that covered the different types of knowledge presented by Mora and Parga (2008) in what is known as the Teaching Knowledge Curriculum Content (CDCC) is a knowledge that emerges of the interaction and integration of disciplinary knowledge, the Historical Epistemology, Context awareness and Knowledge Psicopedagógico School, each of these are considered essential for the inclusion of the components of the environmental dimension raised by Caride and Meira (2001) who established as pillars of environmental education, the economic, political, social, cultural, ethical and ecological.

Thus was taken into account for determining documents such as the Institutional Development Plan 2009-2013, Mission and Vision of both the Faculty of Science and Technology, and the Department of Chemistry, on the other hand analyze the syllabus of the spaces academic curriculum offered by the program where they possibly had determined the environmental dimension immersed in one of the components that are cited throughout the investigation. These results will allow the design of a curriculum that includes the environmental dimension in the training of teachers of chemistry.

Key words: Environmental Dimension, Knowledge Educational Curriculum Content, and preservice Teacher.

\section{Introducción}

El Ministerio de Educación Nacional y el Ministerio de Medio Ambiente formularon e implementaron lo que hoy se conoce como la política de educación ambiental (SINA, 2002), en la cual se establece la necesidad de incorporar los aspectos ambientales en todos los niveles escolares; es de allí de donde surge la necesidad de la formación inicial y continua del docente de Química que incluya aspectos de la dimensión ambiental en esta área y por lo tanto el diseño de currículos que permitan incluir los componentes de la Dimensión Ambiental en los diferentes programas que ofrecen las universidades, con especial énfasis en aquellos que tienen que ver con la formación inicial de docentes de ciencias (SINA, 2002) y en particular como en la formación de profesores de química se aborda este, ya que lo ambiental aparece como un campo de problematización del conocimiento, que induce un proceso de internalización de ciertos principios y consideraciones ambientales dentro de los paradigmas tradicionales de las ciencias (Leff,1986). 
Bio-grafia Escritos sobre La Biología y su Enseñanza.

Edición Extra-Ordinaria. ISSN 2027-1034 P. p 87 - 94

Memorias del VII Encuentro Nacional de Experiencias en la Enseñanza de la

Biología y la Educación Ambiental y II Congreso Nacional de Investigación en la

Enseñanza de la Biología

De acuerdo con lo anterior las instituciones de educación superior deben liderar este proceso de formación de docentes teniendo en cuenta los objetivos planteados en la política de educación ambiental, entre los cuales se propone formular estrategias tales como incluir la dimensión ambiental en los currículos, dando prioridad a la formación, la actualización y el perfeccionamiento de docentes (SINA, 2002) para permitir de esta manera incorporar la educación ambiental como eje transversal en los planes, programas, proyectos y otros, que se generen en el sector ambiental y en el sector educativo, a partir de la ambientalización o inclusión de la dimensión ambiental, ya que esta se constituye como respuesta educativa posible ante la actual crisis ambiental planetaria (Puerto, Mora y Parga, 2011). Es de allí donde surge la necesidad de analizar como ha sido incluida la dimensión ambiental en el currículo de la formación docente, en este caso del profesor de química, donde sin impartir una asignatura de tipo ecológico, este docente debe relacionar las problemáticas ambientales y todo lo que éstas conllevan desde lo económico, moral, político y social a sus prácticas de clase visualizadas desde los diferentes tipos de conocimiento como los planteados por Mora y Parga (2008) a partir del CDCC.

Es por esto que para educar en lo ambiental se requiere del diálogo permanente entre todas las especialidades, todas las perspectivas y todos los puntos de vista (SINA, 2002) conocido también como dialogo de saberes (Leff,1986) en este caso, es por ello que nos interesa la formación de profesores de química, por ello se analizo la formación del docente en química, en la Universidad Pedagógica Nacional donde se realizo un estudio del programa curricular en la que su misión es la formación del profesorado en química con la intensión de identificar como éste programa involucra lo establecido por la política de Educación Ambiental y la ONU en busca de la implementación de la dimensión ambiental: que involucra aspectos políticos, culturales, educativos y sociales, teniendo en cuenta que la química en general es considerada por algunos como la ciencia de los conflictos ambientales ligada a la industrialización y para otros la fuente de las respuestas a la crisis ambiental (Puerto, Mora y Parga 2011) que se presentan en la actualidad. A raíz de lo anterior surge la necesidad de analizar si los programas curriculares que forman profesores de ciencias, entre ellas la química, tienen incluida la dimensión ambiental en sus currículos e indagar como el egresado en licenciatura en química de la Universidad Pedagógica Nacional está dando respuesta a las problemáticas ambientales, tomando como punto de partida su disciplina en este caso la docencia de la química a partir de la inclusión de la dimensión ambiental.

\section{Metodología}

En la actualidad se evidencia una distancia entre la educación ambiental y la educación científica la cual se puede visualizar en la estructura de los currículos de formación de docentes de ciencias y de química, donde no existe una 
Bio-grafia Escritos sobre La Biología y su Enseñanza.

Edición Extra-Ordinaria. ISSN 2027-1034 P. p 87 - 94

Memorias del VII Encuentro Nacional de Experiencias en la Enseñanza de la

Biología y la Educación Ambiental y II Congreso Nacional de Investigación en la

Enseñanza de la Biología

integración entre las dos, aunque vale la pena preguntarnos si ¿éstas dos deberían estar integradas o no?, lo que si es claro es que estamos de acuerdo en que hay una necesidad de incluir la dimensión ambiental la cual se enmarca desde lo legal dándole la importancia suficiente para que desde cualquier programa de formación profesional, en este caso programas de formación de licenciados en química, se genere el puente conector entre el desarrollo sustentable y por ende la ambientalización curricular generando una respuesta óptima de las universidades encargadas de la formación del docente hacia las problemáticas ambientales que aquejan actualmente al mundo.

A partir de las cumbres realizadas por la ONU donde se establece la necesidad de la ambientalización del currículo, de una manera diferente a la imposición de una asignatura de carácter ecológico o ambiental y desde lo planteado en los antecedentes surge la siguiente pregunta: ¿Qué caracteriza la dimensión ambiental en los programas de formación inicial del profesorado de química en las universidades de Bogotá? Esta pregunta se hace ya que consideramos que la formación docente, es tal vez, el punto de partida de un cambio socio-ambiental, por ende es allí donde se debe iniciar el cambio y darle la relevancia que necesita las posibles soluciones a las problemáticas ambientales.

Como supuestos de partida, se considera que:

- Los programas de formación de licenciados se encuentra una incipiente inclusión de la dimensión ambiental.

- La inclusión de la dimensión ambiental esta dada desde una visión poco deseable acerca de la educación ambiental centrada en visiones ecológicas, temas ecológicos, etc.

Estas Hipótesis surgen de la revisión realizada por diferentes investigaciones Nacionales e Internacionales como lo muestra la Red ACES donde se indica de manera incipiente la ambientalización curricular en las universidades hasta el momento de la intervención; por otro lado la insistencia de la ONU por plantear políticas y alternativas en cada cumbre de la Naciones Unidas referentes a aspectos ambientales muestran la debilidad de las universidades en ese proceso que carece del liderazgo de estas. Es así como se plantea la posibilidad de vincular el CDCC (Mora y Parga, 2008) con los pilares de la Dimensión Ambiental (Caride y Meira, 2001), diseñándose y aplicándose la matriz de categorización de la siguiente manera: 
Bio-grafía Escritor sobre la Biología y su Enseñanza.

Edición Extra-Ordinaria. ISSN 2027-1034 P.p 87 - 94

Memorias del VII Encuentro Nacional de Experiencias en la Enseñanza de la

Biología y la Educación Ambiental y 11 Congreso Nacional de Investigación en la

Enseñanza de La Biología

Conocimiento Disciplinar y Creencias (Dis) Esta categoría se define como los conocimientos-creencias del profesorado de química en relación con la educación ambiental, pretendiendo que los profesores se valgan del conocimiento disciplinar de la química con una inclusión de la dimensión ambiental, que visualiza algunos o todos los componentes de la dimensión a trabajar, sin olvidar que el horizonte del conocimiento disciplinar es "comprender la materia" (Parga y Mora, 2008).

Conocimiento Histórico-Epistemológico y Creencias (HyE) El conocimiento Histórico-Epistemológico de los conceptos, teorías y paradigmas se convierten en elemento esencial para determinar la relación entre lo disciplinar y la dimensión ambiental, teniendo en cuenta los mecanismos de producción de conocimiento, los obstáculos epistemológicos, revoluciones científicas y experimentos cruciales (Parga y Mora, 2008) que contribuyan a la imagen de la química en aspectos ambientales.

\begin{tabular}{|c|c|}
\hline DIMENSIÓN & DESCRIPCIÓN E ITEMS \\
\hline $\begin{array}{l}\text { Ecológica- } \\
\text { Tecnocientífica } \\
\text { (DETC). }\end{array}$ & $\begin{array}{l}\text { DETC-PD, busca regularidades y certezas, por ello la ciencia es positivista disciplinar; } \\
\text { se caracterizaría mediante estos indicadores: } \\
\text { (Dis). Teniendo en cuenta los aspectos interdisciplinares y transdisciplinares del campo } \\
\text { ecológico, es decir de la relación entre elementos bióticos y abióticos, unidas a una } \\
\text { visión de la realidad química sustentada en teorías, consideradas verdaderas. } \\
\text { (HyE).Teniendo en cuenta los desarrollos de la historiografía de la química que es vista } \\
\text { desde el enfoque de los mismos científicos y desvinculada de las demás disciplinas, } \\
\text { siendo lo fundamental de la historia, los aspectos internalistas (lógica) entre ellas la } \\
\text { ecológica. } \\
\text { (Con). Aquí lo ecológico es anecdótico y no hace parte de los currículos como una } \\
\text { materia más, quizás es un capítulo de alguna materia y está desvinculado de las } \\
\text { realidades sistémicas y de las interacciones bióticas y abióticas del entorno del medio } \\
\text { educativo. } \\
\text { (PsP). Se caracteriza por una visión analítica de las disciplinas que conforman lo } \\
\text { ecológico articuladas a visiones conductistas del aprendizaje y de las formas } \\
\text { pedagógicas transmisionistas donde lo fundamental de los contenidos son propios de la } \\
\text { química del siglo 19, por lo tanto, la enseñanza no tiene en cuenta aspectos } \\
\text { relacionados con las controversias generadas por la ciencia, sus riegos, sus } \\
\text { incertidumbres y sus conflictos, entre otros. } \\
\text { DETC-CS, intenta explicar la complejidad desde la ciencia social crítica, tiene un } \\
\text { enfoque interdisciplinar, transdisciplinar, el dialogo de saberes y el pensamiento crítico; } \\
\text { donde busca enseñar mediante temas polémicos, como por ejemplo, las cuestiones } \\
\text { socio científicas (CSC). }\end{array}$ \\
\hline ética (DEt) & $\begin{array}{l}\text { DEt-BA, dado que la ética ecológica busca el posicionamiento complejo que plantea } \\
\text { como fundamental la vida de todo lo existente y la diversidad, más allá del interés por la } \\
\text { permanencia de la vida humana sobre la Tierra y la conservación y perpetuidad de la } \\
\text { vida en sus formas diversas. Los indicadores propuestos intentar evaluar: } \\
\text { (Dis). Se trabaja la relación entre conservación y biodiversidad y las interdependencias } \\
\text { entre lo vivo y lo no vivo. La vida implica un abordaje transdisciplinar. } \\
\text { (HyE). El ser humano hace parte de la naturaleza, está en equilibrio y en armonía con } \\
\text { ella, hace uso de lo que requiere como se plantea en las visiones ancestrales. Pero } \\
\text { puede ocurrir que desde visiones de la ecología profunda y desde la sustentabilidad } \\
\text { ambiental el uso de los recursos naturales y de los ecosistemas solo puede ser } \\
\text { aprovechado dando posibilidades a que el ambiente se recupere y todo esto visto desde } \\
\text { la economía ecológica que respeta la entropía de los sistemas. } \\
\text { (Con). Se critican las nociones tecncientificistas en la solución de problemas dado el } \\
\text { impacto ambiental en los cuales se privilegian los valores de la inmediatez, del } \\
\text { individualismo y del utilitarismo. Se proponen nuevos valores desde la sustentabilidad } \\
\text { ambiental vista desde los países del sur como la solidaridad (cooperación) tolerancia, } \\
\text { (diálogo de saberes) la autonomía (espíritu crítico, y la responsabilidad (trabajo útil } \\
\text { socialmente). } \\
\text { (PsP). Lo fundamental es formar en lo que hace posible la vida (su propagación y } \\
\text { calidad). Se forma para que se respete la vida en relación con la naturaleza; se usa la } \\
\text { ética ecológica de responsabilidad y el principio de precaución y protección (principio del } \\
\text { cuidado) para tratar los temas de deterioro de la naturaleza y sus riesgos; la ecoética } \\
\text { fomenta la educación del consumidor. }\end{array}$ \\
\hline social (DS) & $\begin{array}{l}\text { DS-G tiene como indicadores: } \\
\text { (Dis). Las políticas neoliberales fortalecieron el consumismo y la productividad, por lo } \\
\text { tanto lo social es dependiente de lo económico. }\end{array}$ \\
\hline
\end{tabular}


Bio-grafia Escritos sobre la Biología y su Enseñanza.

Edición Extra-Ordinaria. ISSN 2027-1034 P.p 87 - 94

Memorias del VII Encuentro Nacional de Experiencias en la Enseñanza de la

Biología y la Educación Ambiental y 11 Congreso Nacional de Investigación en la

Enseñanza de la Biología

\begin{tabular}{|c|c|}
\hline & $\begin{array}{l}\text { (HyE). La ciencia y la tecnología se ponen al servicio de la economía del mercado. La } \\
\text { dimensión social es dependiente de lo económico. Hay gran tendencia a valorar las } \\
\text { propuestas del desarrollo sostenible donde el concepto de desarrollo la variable } \\
\text { económica es fundamental. } \\
\text { (Con). Lo social está desprovisto del territorio y lo físico geográfico por lo tanto, prima la } \\
\text { descentralización y lo virtual. } \\
\text { (PsP). Se intenta que los procesos de aprendizaje y los principios formativos sean } \\
\text { estandarizados, por lo tanto, los patrones pedagógicos y didácticos son globales y } \\
\text { universales. }\end{array}$ \\
\hline cultural (DC) & $\begin{array}{l}\text { DC-O, es sabido que una pequeña parte de la población del mundo consume los } \\
\text { recursos naturales de la mayoría, y por lo tanto, hay un exceso en el consumo para } \\
\text { tener una vida de lujo, donde se vive con la idea que "cuanto más es mejor, y de lo mas } \\
\text { rápido y barato, y no de lo de mejor calidad". Los indicadores son: } \\
\text { (Dis). Se exaltan las visiones tecno-científicas productivas, articuladas a una } \\
\text { macroeconomía desarrollista que oculta la gravedad de la crisis ecológica, social, } \\
\text { financiera, económica y del conocimiento contemporáneo. El estilo de vida americano y } \\
\text { europeo (huella ecológica) son modelo de la cultura de la globalización, que muchas } \\
\text { veces no tienen en cuenta, que la huella ecológica de esas formas de vida hacen que si } \\
\text { todos los habitantes persiguieran ese modelo, se requerirían tres planetas Tierra para } \\
\text { satisfacer esas necesidades. } \\
\text { (HyE). A lo largo de la historia de la CyT, las modas y la publicidad a través de los } \\
\text { medios de comunicación incitan a consumir y agotar los recursos naturales, por lo tanto, } \\
\text { han demandado el principio de precaución, generando así muchos de los problemas de } \\
\text { salubridad y ambientales de la actualidad. } \\
\text { (Con). Lo fundamental es la competitividad, las evaluaciones constantes basados en } \\
\text { parámetros estandarizados de calidad, orientados a los desempeños para la } \\
\text { productividad. Todo esto en contravía del principio de calidad como principio de hacer } \\
\text { mejor las cosas y por lo tanto, de hacer las cosas con responsabilidad y precaución. Hay } \\
\text { un individualismo masificado que conduce a la deshumanización, es decir supedita lo } \\
\text { social a lo individual. } \\
\text { (PsP). Se sigue formando a los sujetos para la competitividad y el emprendimiento, son } \\
\text { pedagogías para la competitividad. Se pone a la sociedad en riesgo, en amenaza, en } \\
\text { incertidumbre, con inseguridad ecológica, estrés, envejecimiento prematuro, suicidios, } \\
\text { entre otros. }\end{array}$ \\
\hline económica (DEc) & $\begin{array}{l}\text { Ec-M, en la visión de mercado, la CyT están supeditadas a interpretaciones } \\
\text { economicistas y desarrollistas. Sus indicadores son: } \\
\text { (Dis). Como la ciencia y tenología están al servicio de la economía y la ciencia ha } \\
\text { pasado de ser una institución con una imagen altruista, objetiva, para transformarse en } \\
\text { lo que se conoce como la "big science", que se pone al servicio de la industria para } \\
\text { producir distintos productos demandados para el consumo; por eso, la CyT se vuelven } \\
\text { ideologías y cargadas de un sistema de valores de la economía de mercado y del } \\
\text { crecimiento económico, que la han conducido a la generación de actitudes negativas } \\
\text { hacia la ciencia por su impacto ambiental y económico. } \\
\text { (HyE). Se miran las relaciones que ha habido entre el transito y el papel que de la CyT } \\
\text { en la pre modernidad, modernidad y posmodernidad y sus relaciones con el progreso y } \\
\text { el desarrollo económico en los momentos y los cambios entre el desarrollismo y pos } \\
\text { desarrollismo, enfatizando en los momentos de crisis de la modernidad occidental. } \\
\text { (Con). Hay una tendencia a la uniformización del sistema económico mundial } \\
\text { (globalización de la actividad productiva, homogenización del consumo, etc., para lo cual } \\
\text { los avances tecno científicos han permitido multiplicar la productividad del trabajo por } \\
\text { mas de } 20 \text { veces en el siglo XX, por lo tanto, la conformación de la socio-esfera es } \\
\text { consecuencia directa de la transformación de la biosfera por medio de la tecnosfera, } \\
\text { definida como el conjunto de relaciones del conocimiento científico y la tecnología, } \\
\text { aplicadas para la satisfacción de necesidades humanas, a la manera de una caja de } \\
\text { pandora que lleva de riesgos, y subproductos pocos deseables asociados al progreso } \\
\text { tecno-científico. } \\
\text { (PsP). La pedagogía y la didáctica privilegian una CyT al servicio de la economía, por lo lo } \\
\text { que la ciencia está articulada a la tecnología para producir bienes de consumo. }\end{array}$ \\
\hline
\end{tabular}


Bio-grafia Escritos sobre La Biología y su Enseñanza.

Edición Extra-Ordinaria. ISSN 2027-1034 P. p 87 - 94

Memorias del VII Encuentro Nacional de Experiencias en la Enseñanza de la

Biología y la Educación Ambiental y II Congreso Nacional de Investigación en la

Enseñanza de La Biología

\begin{abstract}
político (DP)
DP-R, hay una necesidad de fomentar acuerdos mundiales a favor de un desarrollo sostenible universal, con los siguientes indicadores:

(Dis). Se muestran la necesidad de pasar de una sociedad representativa a una participativa, para que la sociedad esté al tanto de los riesgos producto de la economía y de los desarrollos tecno científicos, que han puesto en peligro y desestabilidad al planeta Tierra en razón a que la mayoría de las crisis actuales son planetarias y no solo locales.

(HyE). Se requiere tener conciencia del impacto que ha tenido las políticas que han estimulado la productividad y el consumo en pro de generar empleo y que han desconocido su impacto sobre el medio y que han conducido a los grandes problemas ambientales centrado en la contaminación, agotamiento de recursos, perdida de diversidad biológica y cultural y la organización creciente y desordenada.

(Con). Es importante entender que la ciencia y la tecnología han estado atadas a los modelos de desarrollo en un tránsito desde la sociedad tradicional pasando de la moderna a la posmoderna. La sociedad tradicional sustentada en economía estacionaria, la moderna que maximiza el crecimiento económico y las pos moderna que maximiza el bienestar subjetivo.

(PsP). Se requiere una educación en ciencias más humana, social y ambiental, forjadas dentro de una concepción política, democrática, critica y participativa.
\end{abstract}

Conocimiento del Contexto Escolar y Creencias (Con) El conocimiento del contexto escolar juega un papel importante en la educación desde todos sus ámbitos, pero esencialmente cuando se habla de inclusión de la Dimensión Ambiental el docente debe tener conocimiento del contexto escolar en el que se encuentra su estudiante determinando la configuración cultural, política e ideológica de éste (Parga y Mora, 2008).

Conocimiento psicopedagógico (PsP) El conocimiento psicopedagógico se fundamenta en aprender a pensar la materia desde la perspectiva del estudiante (Mora y Parga, 2008) donde este conocimiento juega un papel importante en la inclusión de la Dimensión Ambiental, ya que debe tener en cuenta los modelos de desarrollo y aprendizaje de los estudiantes, además de las concepciones alternativas de estos (Mora y Parga, 2008) los cuales se convierten en vitales para transversalidad de la Dimensión Ambiental.

\title{
Resultados y Análisis de Resultados Parciales
}

Para el análisis de los syllabus del programa se tuvo en cuenta la anterior matriz de categorización utilizando el análisis documental a manera de parafraseo, que especificara la posible inclusión de la Dimensión Ambiental en el programa; como se muestra a continuación, dando paso a la fase numero 2, donde se pretende corroborar esta información mediante encuestas y entrevistas a desarrollar a los docentes encargados de dirigir los espacios académicos en los cuales se encontró aspectos ambientales:

\begin{tabular}{|c|c|c|c|c|}
\hline Espacio Académico & $\begin{array}{l}\text { Item Donde Fue } \\
\text { Encontrado }\end{array}$ & Palabra o Frase & Categoría & Subcategoría \\
\hline \multirow{7}{*}{ GEOCIENCIAS } & \multirow{7}{*}{$\begin{array}{c}\text { Justificación Espacio } \\
\text { Académico }\end{array}$} & \multirow{7}{*}{$\begin{array}{c}\text { Acontecimientos } \\
\text { Naturales }\end{array}$} & \multirow{3}{*}{ I.D.S. } & Económica \\
\hline & & & & Ecológica \\
\hline & & & & Social \\
\hline & & & \multirow[t]{3}{*}{ I.D.A. } & Cultural \\
\hline & & & & Política \\
\hline & & & & Ética \\
\hline & & & C.D.C.C. & Disciplinar \\
\hline
\end{tabular}


Bio-grafía Escritos sobre la Biología y su Enseñanza.

Edición Extra-Ordinaria. ISSN 2027-1034 P.p 87 - 94

Memorias del VII Encuentro Nacional de Experiencias en la Enseñanza de la

Biología y la Educación Ambiental y II Congreso Nacional de Investigación en La Enseñanza de la Biología

\begin{tabular}{|c|c|c|c|c|}
\hline & & & & Contexto Escolar \\
\hline & & & & Psicopedagógica \\
\hline Espacio Académico & $\begin{array}{l}\text { Item Donde Fue } \\
\text { Encontrado }\end{array}$ & Palabra o Frase & Categoría & Subcategoría \\
\hline \multirow{10}{*}{ ED. AMBIENTAL } & \multirow{10}{*}{$\begin{array}{c}\text { Justificación Espacio } \\
\text { Académico }\end{array}$} & \multirow{10}{*}{$\begin{array}{l}\text { Formar seres } \\
\text { humanos críticos, } \\
\text { reflexivos y } \\
\text { responsables con el } \\
\text { ambiente }\end{array}$} & \multirow[t]{3}{*}{ I.D.S. } & Económica \\
\hline & & & & Ecológica \\
\hline & & & & Social \\
\hline & & & \multirow[t]{3}{*}{ I.D.A. } & Cultural \\
\hline & & & & Política \\
\hline & & & & Ética \\
\hline & & & \multirow[t]{4}{*}{ C.D.C.C. } & Disciplinar \\
\hline & & & & HyE \\
\hline & & & & Contexto Escolar \\
\hline & & & & Psicopedagógica \\
\hline
\end{tabular}

\section{Conclusiones}

Debido a que el aula es un escenario importante de cambio, se plantea la propuesta de relacionar los diversos conocimientos planteados en el CDCC (Mora y Parga, 2008) y los polos dialecticos ambientales planteados por Caride y Meira en el 2001, como la respuesta a la ambientalización curricular que deberían manejar las universidades encargadas de la formación de profesores en química, ya que esta propuesta no se enfoca netamente en lo disciplinar, o directamente en lo ambiental, sino que por el contrario plantea una relación directa entre los dos ejes principales permitiendo una transversalización critica y constructiva a la respuesta que se pide por parte de las universidades en busca de posibles soluciones a la crisis ambiental que hoy en día afronta la sociedad.

Es por esto que la propuesta que en este trabajo se encuentra desarrollando aporta de manera significativa a la enseñanza de la química y la educación ambiental de manera integrada ya que tiene en cuenta ambos componentes, dándole la misma relevancia. Por ende después del análisis documental se pretende corroborar la información recolectada, validar y diseñar la propuesta curricular que incluya la enseñanza de la química y la dimensión ambiental de manera equilibrada y complementaria.

\section{Bibliografía}

Caride J. y Meira, P. (2001) Educación Ambiental y Desarrollo Humano. Barcelona, Ariel Educación.

Leff, E. (1986). "Perspectiva ambiental del desarrollo del conocimiento" en Los problemas del conocimiento y la perspectiva ambiental del desarrollo. Ed. Siglo XXI. México.

Mora W. M. Y Parga D. L. (2008), El Conocimiento Didáctico del Contenido en Química: integración de las Tramas de contenido / histórico - epistemológicas 
Bio-grafia Escritos sobre la Biología y su Enseñanza.

Edición Extra-Ordinaria. ISSN 2027-1034 P.p 87 - 94

Memorias del VII Encuentro Nacional de Experiencias en la Enseñanza de la Biología y la Educación Ambiental y 11 Congreso Nacional de Investigación en la

$$
\text { Enseñanza de la Biología }
$$

con las Tramas de Contexto / Aprendizaje. En: Revista Tecne, Episteme y didaxis: TED. No. 24. Pag $56-81$.

Puerto, L. Mora, W. Parga, D. (2010). Ambientalización Curricular de los programas de enseñanza química en la educación media: un estudio de caso. Universidad Pedagógica Nacional. Tesis de Maestría.

SINA, (2002) Política de Educación Ambiental. Ministerio de Medio Ambiente. Bogotá, Colombia. 